\title{
Assessing the Validity of Transient Photovoltage Measurements and Analysis for Organic Solar Cells
}

\author{
Sebastian Wood, James C. Blakesley, and Fernando A. Castro \\ National Physical Laboratory, Hampton Road, Teddington TW11 OLW, United Kingdom
}

(Received 21 August 2017; revised manuscript received 27 March 2018; published 24 August 2018)

\begin{abstract}
Transient photovoltage and photocurrent responses of organic solar cells provide valuable information regarding device performance and charge-carrier dynamics. However, the techniques used for analyzing these data have only been shown to be valid in a limited set of circumstances. Here we make use of a quasithree-dimensional model to simulate these measurements in order to quantitatively evaluate the validity of the parameters extracted. With the experimentalist in mind, we produce a set of criteria that can be easily tested and demonstrate a good degree of generality for different sample parameters. This approach allows us to explore the use of "large perturbation" conditions in order to increase the signal-to-noise ratio and speed of the measurement. We propose the introduction of an "effective fixed capacitance," which is shown to significantly reduce the uncertainty associated with the deviation from the small-perturbation regime.
\end{abstract}

DOI: 10.1103/PhysRevApplied.10.024038

\section{INTRODUCTION}

Transient-photovoltage (TPV) and transient-photocurrent (TPC) response measurements are established tools for analyzing the charge-carrier dynamics of photovoltaic samples, which contribute to progress towards highefficiency solar cells [1]. Throughout the last decade, these techniques have been applied extensively to excitonic and organic photovoltaics, where ongoing research holds out the promise of low-cost, printable solar cells [2-9]. Since the first application of TPV measurements to organic photovoltaics, the materials, architectures, and physical dimensions of samples have changed considerably as the technology has advanced towards commercialization. However, it has been widely assumed that this conventional approach to TPV has remained valid. This study assesses the validity of TPV measurements (along with related TPC measurements) and their analysis for organic solar cells by using a computational simulation to explore the interpretation of experimental results.

The conventional method for performing and analyzing TPV measurements on organic photovoltaics has been described by Shuttle et al. [10], building on the more established use of the technique for dye-sensitized solar cells. In this measurement, the voltage across a photovoltaic device is measured and the time-resolved response to a nanosecond optical excitation pulse is recorded. Since the photovoltage is related to the photogenerated chargecarrier density in the device, the decay of the photovoltage

\footnotetext{
*sebastian.wood@npl.co.uk
}

transient provides a measure of charge recombination rate. An additional continuous bias light with adjustable intensity provides a means of controlling the charge-carrier density in the device such that the relationship between the charge-carrier lifetime and charge-carrier density can be elucidated. In the conventional method, a number of assumptions are required in order to extract quantitative results from the experiment. In this manuscript we consider three major assumptions: (1) complete charge extraction in the TPC measurement with no bias light; (2) the smallperturbation approximation in TPV measurement; and (3) the differential capacitance method for approximating charge-carrier density.

These assumptions were shown to be valid in the case of small $\left(4.5 \mathrm{~mm}^{2}\right)$ devices comprising blended poly $(3-$ hexylthiophene) and [6,6]-phenyl- $\mathrm{C}_{61}$-butyric acid methyl ester (P3HT:PCBM), where transient absorption spectroscopy provides a complementary experimental measurement of the P3HT polaron population in response to photoexcitation [8]. However, the most commercially relevant organic photovoltaic devices use alternative device architectures, have much larger active areas, and often make use of novel organic semiconductor materials, which yield higher power conversion efficiencies than P3HT [1114]. In these cases, it is not clear whether the same assumptions are valid and how sensitive these assumptions are to the measurement conditions. This places a serious limitation on the use of TPV measurements for the characterization of organic solar cells. Some level of experimental verification of the results is possible in certain cases (e.g., using transient absorption spectroscopy), but such techniques are 
not widely available and somewhat undermine the value of TPV as a quick and simple measurement. Additionally, the technique is typically performed in the small-perturbation regime using low-intensity excitation and resulting in a small signal. Various criteria are used by experimentalists in an effort to satisfy this approximation, such as ensuring that the transient voltage peak is less than $5 \%$ of the open-circuit voltage or keeping the voltage peak below $10 \mathrm{mV}[4,15]$. However, there is no clear justification for these values in most cases. A stronger signal and faster data acquisition can be achieved by relaxing this constraint but it remains unclear to what extent this compromises the validity of the measurement [16]. Furthermore, conventional interpretation of TPV and TPC measurements assumes an idealized one-dimensional device structure in which lateral conduction and other effects related to device geometry are neglected. While it is possible to prepare small devices to minimize the impact of device geometry and dimension, the application of the technique to inspection of consumer products necessitates the validation of the technique on larger devices with cell widths of the order of $1 \mathrm{~cm}$ or greater.

This work seeks to address these concerns regarding the accuracy of charge-carrier-dynamic parameters derived from TPV measurements by developing a set of validity criteria, which can be assessed during the experiment itself without the need for additional equipment or complex measurements. To achieve this, we perform a computational simulation of TPV measurements enabling the rapid exploration of a wide range of parameters to explore the range of conditions under which the TPV method fails. This enables a direct comparison between input values describing the simulated device and the output values derived using the established methods for analyzing TPV data. Comparing these input (expected) and output (extracted) values reveals the limitations of TPV analysis, and our simulation allows us to probe the origins of these discrepancies and propose experimental guidelines to ensure accurate results. This approach is represented diagrammatically in Fig. 1.

\section{EXPERIMENTAL METHODS}

Samples are prepared in an inverted architecture, ITO/ $\mathrm{ZnO} /$ polymer:fullerene $/ \mathrm{MoO}_{x} / \mathrm{Ag}$, with an active area of $1 \mathrm{~cm}^{2}$, according to the method described previously [17]. The chemical structures and optoelectronic properties of the polymer and fullerene materials have been discussed previously under the names "PBTZT-stat-BDTT-8" and "PV-A600," respectively [18].

Current-voltage characteristics under 1 Sun $(1000$ $\mathrm{W} \mathrm{m}^{-2}$, AM1.5G spectrum) illumination are measured using a Newport 91195A solar simulator and a Keithley 2604B SourceMeter. Photocurrent and photovoltage transient responses are measured by connecting the sample to a Tektronix DPO4104B oscilloscope with $50 \Omega$ and $1 \mathrm{M} \Omega$ input impedance, respectively. The pulsed optical excitation is provided by a Sirah PrecisionScan dye laser with $635 \mathrm{~nm}$ wavelength and $<10 \mathrm{~ns}$ pulse duration, delivering pulses with an energy of $3 \mathrm{~mJ}$ at $10 \mathrm{~Hz}$, and the pulse fluence at the sample is reduced by an approximate factor of 1000. The beam is expanded to excite the whole active area of the device with an approximately Gaussian beam profile (FWHM $16.0 \mathrm{~mm}$ ). Bias light is provided by an OSLON80 9+ PowerStar LED array with a peak wavelength of $617 \mathrm{~nm}$ driven with a constant current up to $1 \mathrm{~A}$. For this device, the maximum bias-light intensity gives a current density equivalent to that observed at around 0.75 Sun.

In order to ensure the widespread applicability of the results considered in this work, a generalized computational model of the experiment was developed in-house using the MATLAB software package [19]. This avoided issues of instrumentation or device-specific effects and enabled virtual experiments to be performed on a wider parameter space of device and measurement conditions than was feasible experimentally.

The computational model developed for this work is a transient finite-element model (FEM) of a thin film sandwiched between two parallel electrodes. Optical excitation is modeled by the spatially uniform generation of charge within the active layer. Lateral conduction is assumed to take place only within the electrodes, which are modeled as ohmic sheets with uniform sheet resistivity, and defined geometry and dimensions. Vertical charge transport through the film between the electrodes is modeled by a one-diode equivalent-circuit model that has been modified to reproduce the physics of power-law recombination rates assumed in the conventional interpretation of TPC and TPV results. Thus, the implementation of the FEM scheme reduces a simulated experiment to a distributed equivalent-circuit model whose output converges to the conventional analytical interpretation under ideal measurement conditions and device geometries. A more complete description of the model is given in Appendix A.

The unknown model parameter values are tuned to create a simulated device with properties that fit experimental measurements for a physical sample. Figure 2 compares the current-voltage characteristics of the experimental and simulated devices demonstrating that a good fit is possible, which gives a close correspondence with the experimental device performance parameters: short-circuit current $\left(J_{\mathrm{SC}}\right)$, open-circuit voltage $\left(V_{\mathrm{OC}}\right)$, fill factor $(\mathrm{FF})$, and power conversion efficiency (PCE) at 1 Sun.

Table I lists the device parameters used in the simulation to reproduce the experimental results. The values for active layer thickness and active area are taken from those of the experimental sample; similarly, the indium tin oxide (ITO) and Ag electrode conductances are taken from quoted values for the materials. A relative permittivity value of 3.5 


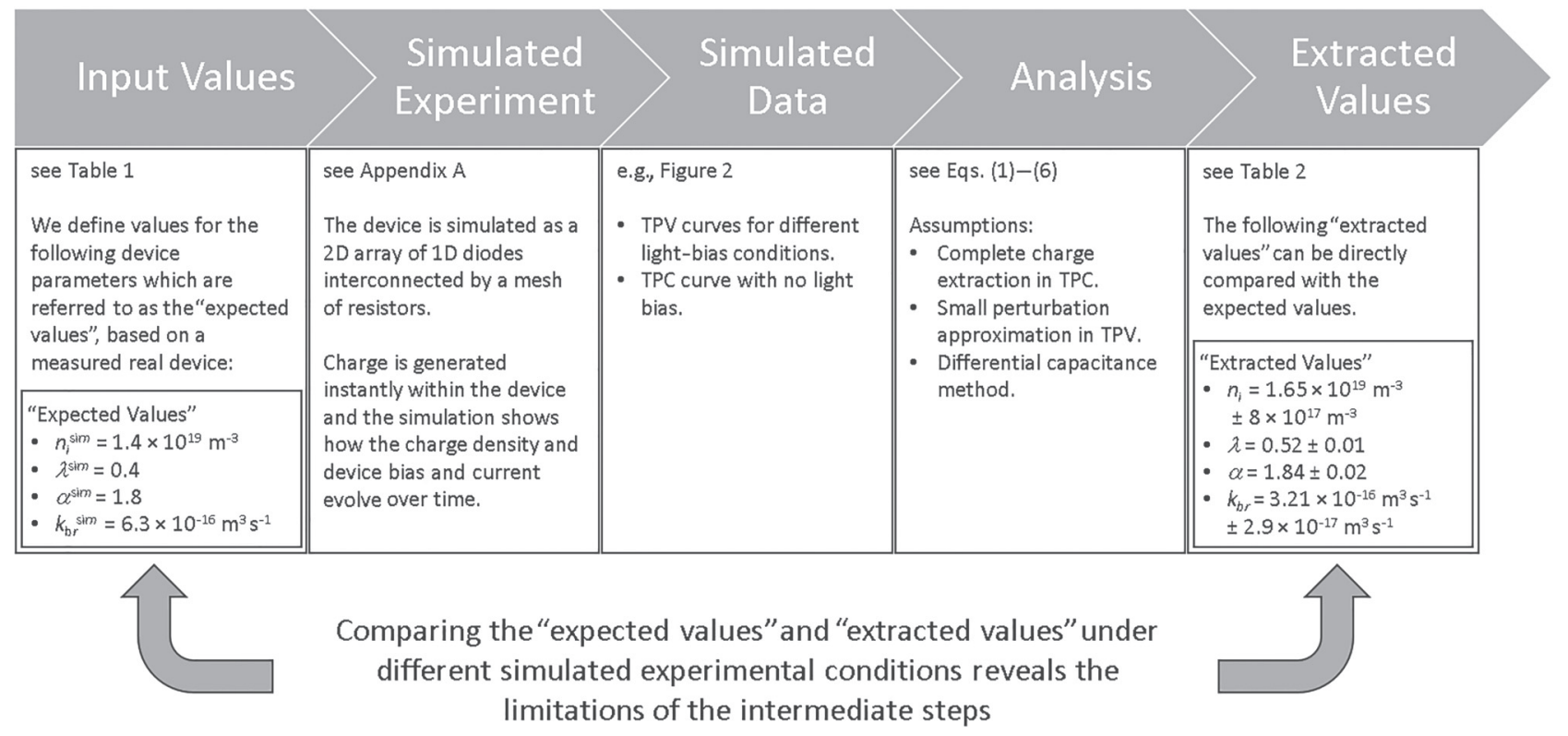

FIG. 1. Flow diagram describing the work conducted in this study. Input parameters are put into a simulated TPV experiment resulting in a simulated data set. These data are then fed into the established analytical methods used for extracting values from TPV results. If the data analysis is valid we expect these extracted values to match the expected values (corresponding directly to the input parameters). In this study we use the simulation to explore the experimental parameter space and identify the regime for which the extracted values match the expected values.

is taken as a typical value for an organic semiconductor device [20]. The other values are chosen iteratively to give a good match between simulated and experimental current-voltage characteristics using values within the expected range for each parameter. The light intensity is calculated by assuming a linear relationship with the charge-carrier density generation rate in the simulation, where 1 Sun is roughly equivalent to charge generation at $3 \times 10^{27} \mathrm{~s}^{-1} \mathrm{~m}^{-3}$ based on the solar irradiance integrated over the visible range and assuming an external quantum efficiency (EQE) of $75 \%$ for visible wavelengths (a typical

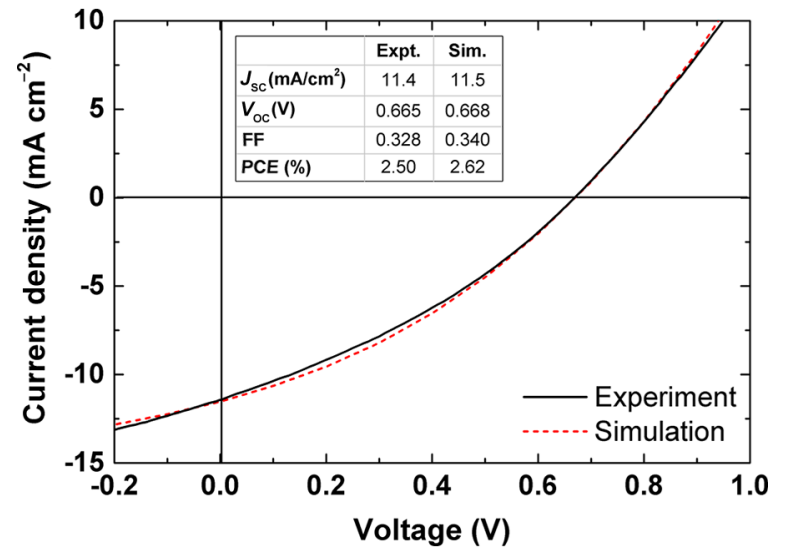

FIG. 2. Comparison of current-voltage characteristics for experimental and simulated devices measured under an illumination of 1 Sun. The inset table compares device parameters indicated by these characteristics. value for a good organic solar cell) [21]. Here the model considers a laser excitation pulse with duration $5 \mathrm{~ns}$ and an effective pulse fluence of approximately $100 \mu \mathrm{J} \mathrm{m}^{-2}$.

In addition to the steady-state current-voltage characteristics, the model is also able to simulate the transient photovoltage and photocurrent response of the device. These results are compared with equivalent experimental results in Fig. 3 showing TPV signals for a range of biaslight intensities up to 0.75 Sun and TPC signals with no bias light. The magnitudes of the experimental voltage offsets, peak heights, and rise and decay times are all found to

TABLE I. Device simulation input parameters.

\begin{tabular}{lll}
\hline \hline Parameter & Symbol & Value \\
\hline Active-layer thickness & $d$ & $300 \mathrm{~nm}$ \\
Active-layer area & $A$ & $1 \mathrm{~cm}^{2}$ \\
Active-layer relative permittivity & $\varepsilon_{r}$ & 3.5 \\
ITO electrode sheet resistance & $R_{0}$ & $15 \Omega \square^{-1}$ \\
Ag electrode sheet resistance & $R_{1}$ & $0.1 \Omega \square^{-1}$ \\
Shunt resistivity & $R_{\mathrm{sh}}$ & $1 \times 10^{8} \Omega \mathrm{m}$ \\
Effective charge-carrier & $\mu$ & $5 \times 10^{-8} \mathrm{~m}^{2} \mathrm{~V}^{-1} \mathrm{~s}^{-1}$ \\
$\quad$ mobility & & \\
Intrinsic charge-carrier density & $n_{i}^{\text {sim }}$ & $1.4 \times 10^{19} \mathrm{~m}^{-3}$ \\
Charge-carrier & $\lambda^{\operatorname{sim}}+1$ & 1.4 \\
$\quad$ recombination order & & \\
Carrier-density ideality factor & $\alpha^{\mathrm{sim}}$ & 1.8 \\
Effective bimolecular & $k_{\mathrm{br}}^{\text {sim }}$ & $6.3 \times 10^{-16} \mathrm{~m}^{3} \mathrm{~s}^{-1}$ \\
recombination rate & & \\
\hline \hline
\end{tabular}



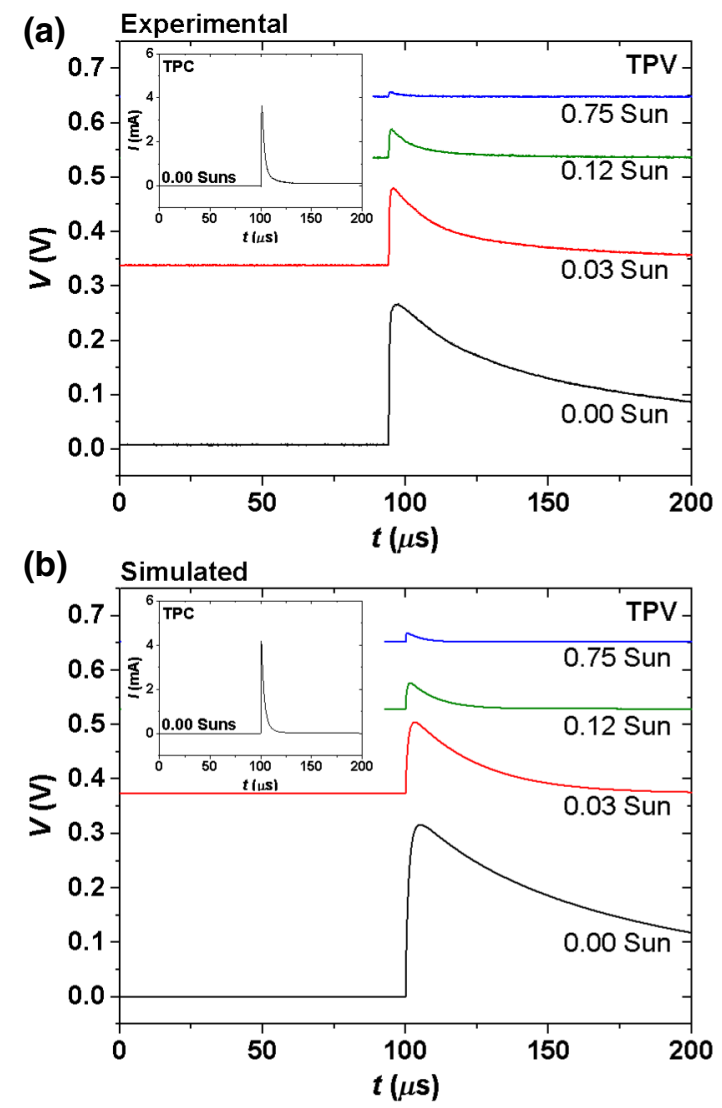

FIG. 3. Comparison of (a) experimental and (b) simulated voltage transients with bias-light intensities equivalent to $0.00,0.03$, 0.12 , and 0.75 Sun. Insets show photocurrent transients at 0.00 Sun light bias.

be reproduced realistically by the simulation. In fact, based only on the experimental $J-V$ curves, there are multiple sets of parameter values that can provide a good fit, but considering the transient response too removes this ambiguity. We note that, while it is important to establish that the simulation produces a realistic representation of an experimental device, we do not require it to perfectly match any specific sample. As a result, the minor differences between the experimental and simulated results compared in Figs. 2 and 3 are not critical for this study.

\section{RESULTS AND DISCUSSION}

Throughout the remainder of this article, all of the results shown are taken from computational simulations of the TPV experiment (as described in Sec. II) rather than experimental results. The advantage of this approach is that the input parameters of the model are clearly defined and the charge-carrier density in the device is known from the simulation data. This provides a means of quantitatively assessing and diagnosing discrepancies in the extracted values derived using the conventional analysis techniques and the expected values taken directly from the simulation.
The conventional TPV measurement seeks to determine the rate law of charge-carrier density decay in a device by considering the decay rate as a function of charge-carrier density. These parameters are not directly measurable, but the TPV signal provides an indirect measure (requiring a number of simplifying assumptions). In the small-perturbation regime, where the additional charge density generated by the excitation laser pulse is much smaller than the background charge-carrier density, the lifetime of the charge-carrier density perturbation $\tau_{\Delta n}$ is equal to the lifetime of the TPV signal $\tau_{\Delta V}$, and both follow the same single exponential decay function given in Eq. (1). To measure this decay for different charge-carrier densities $n$, a separate bias light with variable intensity is used to generate a range of background charge levels resulting in different open-circuit voltages $V_{\mathrm{OC}}$. From these measurements, the TPV decay lifetime is found to follow an exponential dependence on $V_{\mathrm{OC}}$, as in Eq. (2). In order to evaluate the charge-carrier density corresponding to each value of $V_{\mathrm{OC}}$, we make use of the differential capacitance $C$ given in Eq. (3). In this equation, we already know $\Delta V_{0}$ from the fitted amplitude of the TPV signal, but finding $\Delta Q$ requires an additional measurement of the TPC signal (with the same experimental conditions as TPV but with low input impedance on the oscilloscope and no bias illumination). Assuming efficient charge extraction, the integrated TPC signal provides a measure of the total charge generated by the laser pulse $(\Delta Q)$. Empirically, the differential capacitance values are found to follow the exponential dependence on the open-circuit voltage given by Eq. (4), and so the charge-carrier density as a function of $V_{\mathrm{OC}}$ is given by treating the device as a parallel-plate capacitor and integrating with respect to voltage, as in Eq. (5). This process results in a set of values for the TPV decay lifetime at different charge-carrier densities, $\tau_{\Delta V}(n)$, which can be fitted to a power law with order $\lambda$, shown by Eq. (6) [8].

$$
\begin{gathered}
V=\Delta V_{0} \exp \left(\frac{-t}{\tau_{\Delta V}}\right), \\
\tau_{\Delta V}=\tau_{\Delta V 0} \exp \left(-\beta V_{\mathrm{OC}}\right), \\
C=\frac{\Delta Q}{\Delta V_{0}}, \\
C=C_{0} \exp \left(\gamma V_{\mathrm{OC}}\right)+D, \\
n=\frac{1}{A e d} \int_{-\infty}^{V_{\mathrm{OC}}} C_{0} \exp (\gamma V) d V, \\
\tau_{\Delta V}=\tau_{\Delta V 0}\left(\frac{n}{n_{i}}\right)^{-\lambda} .
\end{gathered}
$$

In the small-perturbation regime, $\tau_{\Delta n} \approx \tau_{\Delta V}$ and the charge-carrier recombination has been shown to follow the rate equation $(d n / d t) \propto-n^{1+\lambda}[8]$. 
TABLE II. List of variables used in analysis of TPV measurement data and values extracted from simulated TPV results.

\begin{tabular}{lll}
\hline \hline Variable name & Symbol & Extracted value \\
\hline TPV magnitude & $\Delta V_{0}$ & Various $(\mathrm{V})$ \\
TPV decay time & $\tau_{\Delta V}$ & Various (s) \\
Charge-carrier-density decay time & $\tau_{\Delta n}$ & Various $(\mathrm{s})$ \\
Total charge generated by pulse & $\Delta Q$ & $1.72 \times 10^{-8} \mathrm{C} \pm 1 \times 10^{-9} \mathrm{C}$ \\
TPV decay-time prefactor & $\tau_{\Delta V 0}$ & $1.89 \times 10^{-4} \mathrm{~s} \pm 7 \times 10^{-6} \mathrm{~s}$ \\
TPV decay-time exponential constant & $\square$ & $5.5 \mathrm{~V}^{-1} \pm 0.1 \mathrm{~V}^{-1}$ \\
Differential capacitance & $C$ & See Fig. $4(\mathrm{~b})(\mathrm{F})$ \\
Effective fixed capacitance & $D$ & $6.45 \times 10^{-8} \mathrm{~F} \pm 1.1 \times 10^{-9} \mathrm{~F}$ \\
Exponential capacitance prefactor & $C_{0}$ & $8.30 \times 10^{-10} \mathrm{~F} \pm 3.8 \times 10^{-11} \mathrm{~F}$ \\
Capacitance exponential constant & $\gamma$ & $10.5 \pm 0.1 \mathrm{~V}^{-1}$ \\
Bulk charge-carrier density & $n$ & See Fig. $4(\mathrm{~b})\left(\mathrm{m}^{-3}\right)$ \\
Intrinsic charge-carrier density & $n_{i}$ & $1.65 \times 10^{19} \mathrm{~m}^{-3} \pm 8 \times 10^{17} \mathrm{~m}^{-3}$ \\
TPV decay-time power-law order & $\lambda$ & $0.52 \pm 0.01$ \\
TPV decay-time power-law order $($ neglecting $D)$ & $\lambda^{\prime}$ & $0.70 \pm 0.01$ \\
Carrier density ideality factor $\left(e / 2 \gamma k_{B} T\right)$ & $\alpha$ & $1.84 \pm 0.02$ \\
Effective bimolecular recombination rate $\left(1 / \tau_{\Delta V 0} n_{i}\right)$ & $k_{\mathrm{br}}$ & $3.21 \times 10^{-16} \pm 2.9 \times 10^{-17} \mathrm{~m}^{3} \mathrm{~s}^{-1}$ \\
\hline \hline
\end{tabular}

Note that " $e$ " represents the elementary charge throughout this manuscript and "exp" is used for the exponential function.

Table II identifies the meanings of the variables in these equations along with the values extracted by applying this technique to the simulated TPV data, as illustrated by Fig. 4. Fitting is performed using the LevenbergMarquardt algorithm and the quoted uncertainty values are the standard errors in fitting parameters taken from the covariance matrix. We note that the definitions of these determined parameters mostly do not correspond directly with the input parameters of the simulation and, where they do, the extracted values do not show good agreement with the expected (input) values. This discrepancy is due to the assumptions made in the analysis of the simulated data and its inability to accurately describe the modeled device physics. This work seeks to understand and assess the origins and magnitudes of these discrepancies.

We note that Eq. (4) differs from that presented elsewhere on account of the additional factor, $D$, representing an effective fixed component of the capacitance. Figure 4(b) illustrates the need for this additional factor by comparing the fitted lines obtained with and without including it. We find that the capacitance data cannot be well represented by a simple exponential growth and, furthermore, Fig. 4(c) demonstrates the impact of neglecting the factor $D$ on the value calculated for the power-law order. Specifically, we obtain an extracted value of $\lambda=0.52 \pm 0.01$ when $D$ is included in the fitting, but $\lambda^{\prime}=0.70 \pm 0.01$ when $D$ is neglected. These values are somewhat greater than the expected value of $\lambda^{\text {sim }}=0.4$ used as an input parameter for the simulation; however, the discrepancy is significantly reduced by including the additional factor, $D$. The physical interpretation of the "effective fixed capacitance" is considered in the discussion of the differential capacitance method below.
Performing this analysis of the simulated TPV data results in a value for the charge-carrier recombination order $(\lambda+1)$ of $1.52 \pm 0.01$. This value is within $10 \%$ of the simulated value of 1.4 and could be considered a reasonable approximation; however, the fact that the expected value lies outside the uncertainty range quoted for the extracted value (evaluated from the fitting algorithm) indicates a systematic discrepancy in the analysis arising from the use of an imperfect model. In order to identify and quantify this systematic error we now consider the various assumptions that are employed: (1) complete charge extraction in TPC (evaluating $\Delta Q$ ); (2) the smallperturbation approximation (evaluating $\Delta V_{0}$ and $\tau_{\Delta n}$ ); and (3) the differential capacitance method (evaluating $n$ ).

These assumptions are considered in turn. We find that the first assumption is valid under typical measurement conditions, whereas the second and third are closely related and place constraints on the acceptable pulsed-excitation fluence dependent on the bias-light intensity used.

\section{A. Complete charge extraction in TPC (evaluating $\Delta Q)$}

The differential capacitance is linearly dependent on $\Delta Q$, which is estimated by measuring the TPC response of the device with no applied light bias. In this case, it is expected that the low load $(50 \Omega)$ on the device will result in fast charge extraction such that the loss of charge due to the slower recombination process is minimal. With no bias light, the background charge-carrier density is minimized, which also reduces the impact of charge recombination on the measurement. If the charge extraction current is indeed much greater than the charge recombination current in the device then the integral of the TPC signal gives a 

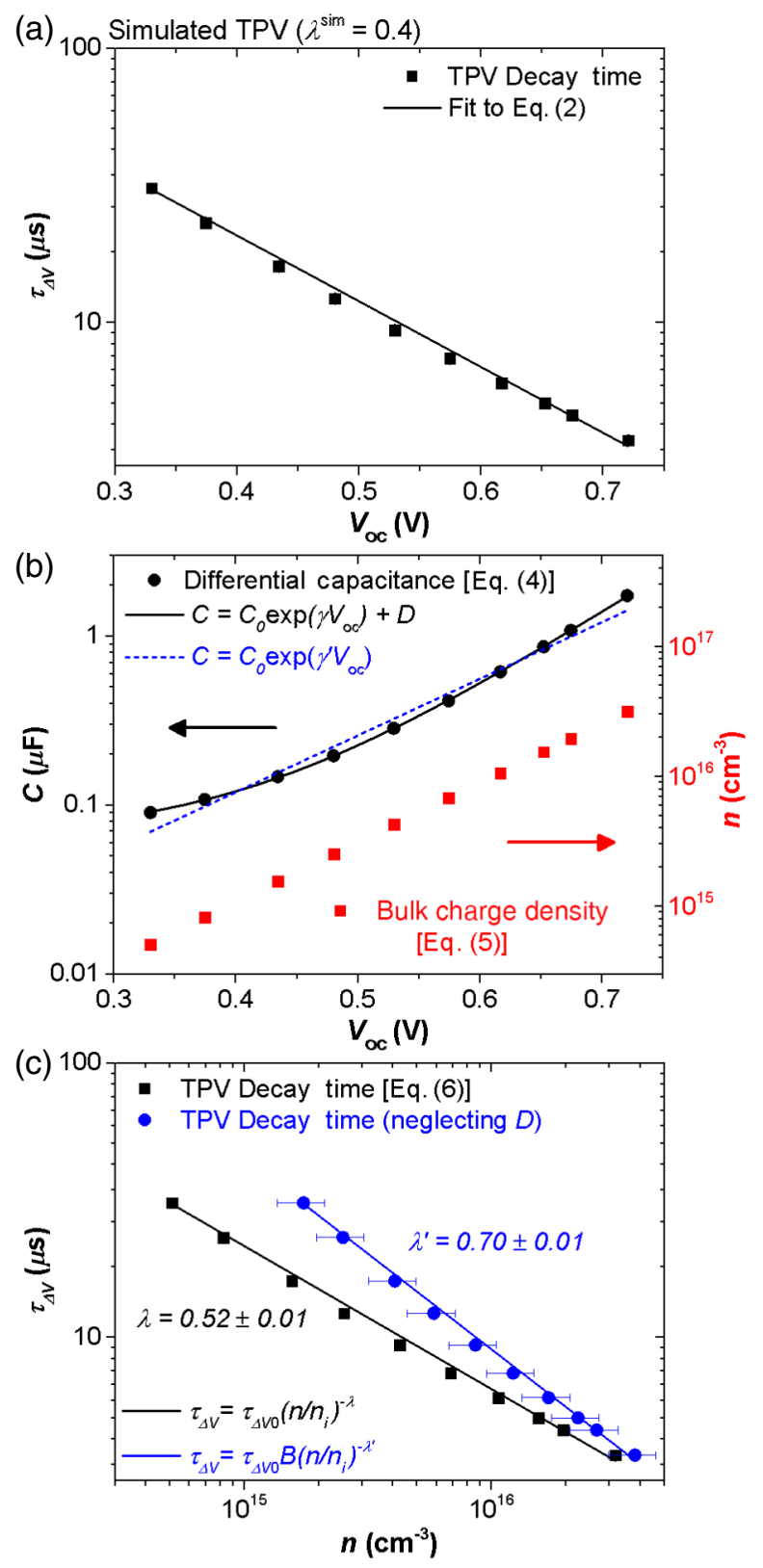

FIG. 4. Results of conventional analysis of simulated TPV results for a device with $\lambda^{\text {sim }}=0.4$, illustrating the need for an effective fixed capacitance, $D$. (a) TPV decay time as a function of $V_{\mathrm{OC}}$ fitted with Eq. (2). (b) Differential capacitance plotted against $V_{\mathrm{OC}}$ fitted with Eq. (4) and comparing fitting with and without the effective fixed capacitance, and bulk charge-carrier density calculated from the differential capacitance via Eq. (5). (c) Plot of TPV decay time against bulk charge-carrier density comparing results extracted with and without the effective fixed capacitance and showing values of $\lambda$ and $\lambda^{\prime}$ obtained by power-law fitting to Eq. (6) in each case.

good measure of the total charge generated in the device by the light pulse. This assumption can be tested by comparing the extracted $\Delta Q$ values, obtained from integrating the simulated TPC signal, with the expected $\Delta Q$

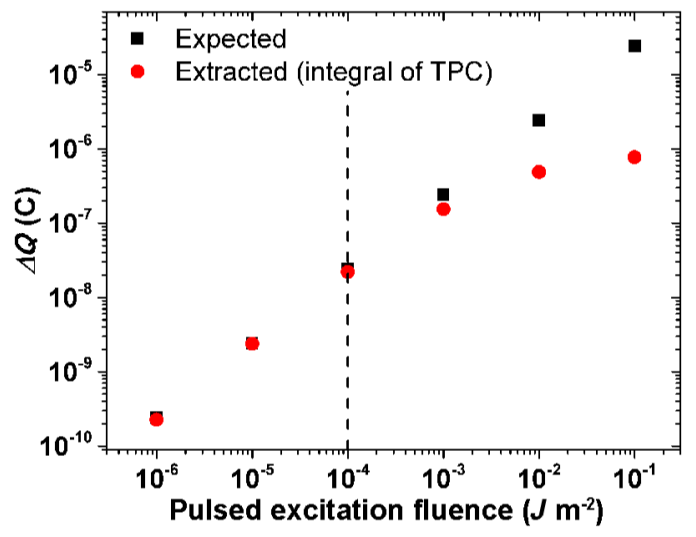

FIG. 5. Plot of charge generated $(\Delta Q)$ as a function of pulsedexcitation fluence comparing the extracted values, obtained by integrating the TPC signal with the expected values of the charge generated in the simulation. The dashed line shows the excitation fluence used for results shown in Figs. 3 and 4.

values, calculated from the input parameters of the simulation (assuming that the photogenerated charge-carrier density is proportional to the excitation fluence). These results are shown in Fig. 5 for a range of excitation-pulse fluences. We find that that these two sets of $\Delta Q$ values are close at low excitation fluences with the value extracted from the TPC signal giving a slight underestimation of the expected value $\left(99 \%\right.$ at $10 \mu \mathrm{J} \mathrm{m}^{-2}$, dropping to $91 \%$ at $100 \mu \mathrm{J} \mathrm{m}^{-2}$ ), but at higher fluences the two sets of values diverge significantly.

For practical purposes, we suggest that the experimentalist assesses the accuracy of the $\Delta Q$ measurement by varying the intensity of the excitation laser pulse using filters with known optical density to ensure that the relationship between $\Delta Q$ and the light intensity is proportional in the regime of the measurement [2]. For example, we might represent the relationship as a power law where $\Delta Q$ $\propto$ (pulse intensity) $z$ and require that $0.95<z<1.05$. This validity criterion is expected to have some dependence on specific measurement and sample conditions. It is also important to note that the maximum permitted intensity of the excitation laser pulse is also subject to considerations related to the small-perturbation regime, discussed below, which are more restrictive.

\section{B. Small-perturbation approximation (evaluating $\Delta V_{0}$ and $\left.\tau_{\Delta n}\right)$}

The small-perturbation approximation is a powerful simplification of the complex dynamics of transient optoelectronic measurements. The basis of this assumption is that the additional charge-carrier density generated in the device as a result of the optical excitation pulse is much smaller than the charge-carrier density already present. This condition can be controlled by using a bias light to increase the background charge-carrier density and by 
minimizing the intensity of the excitation pulse. However, this tends to compromise the signal-to-noise ratio and so presents the experimental challenge of optimizing the measurement while maintaining the validity of the small-perturbation approximation.

In TPV measurements, the small-perturbation approximation enables charge recombination to be considered as a pseudo-first-order decay, where the voltage decay and the charge-carrier density decay share a single decay constant, i.e., $\tau_{\Delta n} \approx \tau_{\Delta V}$. The TPV signal can, therefore, be fitted with a single exponential decay using Eq. (1) from which the decay time $\tau_{\Delta V}$ and amplitude $\Delta V_{0}$ are extracted. The signals typically display a noninstantaneous rise time so, to obtain a meaningful fit, it is necessary to neglect the early-time data points. To do this we apply the criterion that the exponential decay is fitted only to the data after the TPV signal has decayed to $95 \%$ of the peak value (this choice of fitting condition is considered further in Appendix B) and extrapolated back to find $\Delta V_{0}$ at the start of the rise. Figure 6(b) illustrates this fitting for three different cases.

Expected values for $\Delta V_{0}$ based on charge-carrier density values taken from the simulation $\left(n^{\mathrm{sim}}\right)$ can be evaluated from photovoltages given by Eq. (7) and the resulting ratio of the fitted (extracted) values of $\Delta V_{0}$ to the expected values is presented in Fig. 6(a). Values of this ratio vary from 0.2 to 1.2 over the range of measurement conditions considered here with the greatest discrepancies occurring in the regime of low bias intensity (low $V_{\mathrm{OC}}$ ) and low excitation fluence (low $\Delta V_{0}$ ). In the plot, the green area represents the regime where the error in $\Delta V_{0}$ is within $\pm 20 \%$, which may appear to be acceptable as an estimate of $\Delta V_{0}$; however, the value of charge-carrier density extracted from this $\Delta V_{0}$ value is much more sensitive and is considered below.

$$
V=\frac{2 k_{B} T \alpha^{\mathrm{sim}}}{e} \ln \left(\frac{n^{\mathrm{sim}}}{n_{i}^{\mathrm{sim}}}\right) .
$$

In order to understand the origin of the discrepancy in the extracted and expected $\Delta V_{0}$ values, Fig. 6(b) compares simulated TPV signals corresponding to points $A$, $B$, and $C$ in Fig. 6(a) along with the fitted exponential functions (signals normalized to the expected $\Delta V_{0}$ value). It is clear from this comparison that the fitting is most successful when the onset of the transient signal is rapid and becomes progressively less reliable as the voltage rise time increases and the peak becomes rounded. Additional divergence from the model is observed for high excitation intensities and low bias-light intensities. The longer rise time under low bias conditions is believed to be related to lower conductivity, which results in a slower dielectric relaxation time for the photogenerated charge. To ensure consistent data processing, we take the adjusted- $R^{2}$ coefficient of determination as a measure of appropriateness
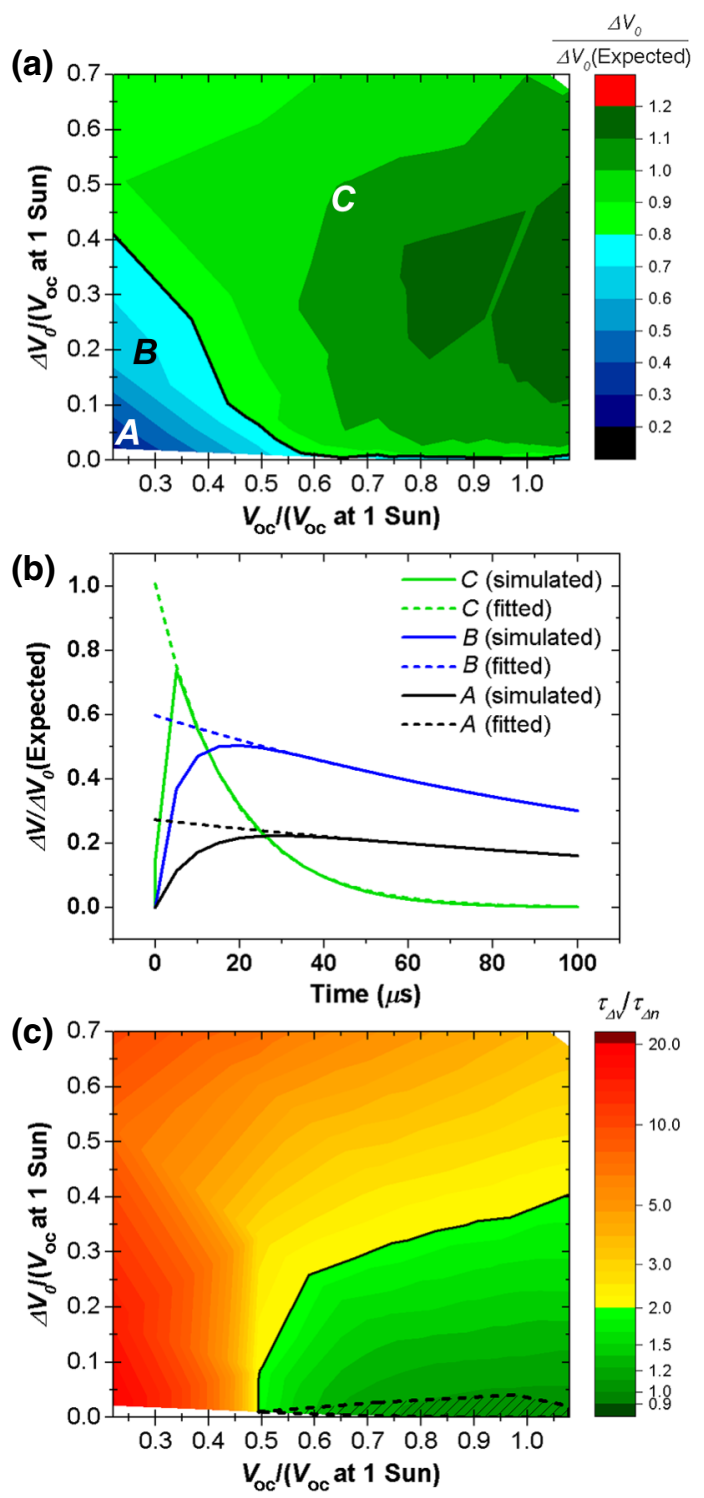

FIG. 6. (a) Color plot showing the ratio $\Delta V_{0} / \Delta V_{0}$ (expected) plotted against $\Delta V_{0} /\left(V_{\mathrm{OC}}\right.$ at $\left.1 \mathrm{Sun}\right)$ and $V_{\mathrm{OC}} /\left(V_{\mathrm{OC}}\right.$ at $\left.1 \mathrm{Sun}\right)$. The green area with black border shows the regime where the measured value is within $\pm 20 \%$ of the expected value. (b) Comparison of simulated TPV transients with their fitted exponentials at regions labeled $A, B$, and $C$ in (a), normalized to the expected value of $\Delta V_{0}$. (c) Color plot showing the ratio $\tau_{\Delta V} / \tau_{\Delta n}$ plotted against $\Delta V_{0} /\left(V_{\mathrm{OC}}\right.$ at $\left.1 \mathrm{Sun}\right)$ and $V_{\mathrm{OC}} /\left(V_{\mathrm{OC}}\right.$ at $\left.1 \mathrm{Sun}\right)$. The green region with black border shows the measurement range where the ratio lies between 0.9 and 2.0. White areas indicate an absence of data points due to limitations of the simulation resolution in extreme regimes. The shaded region indicates the regime satisfying the criterion $\Delta V_{0}<\left(5 \%\right.$ of $\left.V_{0}\right)$.

for the single exponential fitting and reject TPV signals with adjusted- $R^{2}<0.99$. Quantifying the quality of fitting and determining rejection criteria for experimental data are difficult to generalize and so a human assessment is 
required to ensure that the TPV signals are well represented by single exponential decays. In fact, we find that the approximation $\tau_{\Delta n} \approx \tau_{\Delta V}$ is very sensitive to deviation from the small-perturbation regime and likely to present a more restrictive criterion for the validity of the method than deviation from the pseudo-first-order decay fitting itself.

To explore the approximation $\tau_{\Delta n} \approx \tau_{\Delta V}$ we consider the ratio $\tau_{\Delta V} / \tau_{\Delta n}$. Values of $\tau_{\Delta V}$ are extracted by fitting the simulated TPV data with a single exponential decay and the $\tau_{\Delta n}$ values are similarly taken from chargecarrier density transients, which are directly available from the simulation data. Values of this ratio are plotted against $\Delta V_{0}$ and $V_{\mathrm{OC}}$ (normalized to $V_{\mathrm{OC}}$ measured at 1 Sun) in Fig. 6(c). The range plotted corresponds with bias illumination intensities from 0.01 Sun to 2 Sun and pulsed-excitation fluences from roughly $1 \mu \mathrm{J} \mathrm{m}^{-2}$ to $10 \mathrm{~mJ} \mathrm{~m}^{-2}$ with interpolation between data points. Values of $\tau_{\Delta V} / \tau_{\Delta n}$ vary from 0.96 to approximately 20 over this range of parameters indicating that $\tau_{\Delta V}$ is systematically larger than $\tau_{\Delta n}$. The green colored area on the plot indicates the regime where the ratio is $<2$, which corresponds to high light-bias conditions $\left(V_{\mathrm{OC}} / V_{\mathrm{OC}}(1 \mathrm{Sun})>0.5\right)$ and low pulsed-excitation intensity $\left(\Delta V_{0} / V_{\mathrm{OC}}(1\right.$ Sun $\left.)<0.3\right)$ in which the small-perturbation regime could be considered valid. Outside this region, the ratio is very sensitive to the bias-light intensity $\left(V_{\mathrm{OC}}\right)$ and, to a lesser extent, the excitation-pulse intensity $\left(\Delta V_{0}\right)$. The precise form of this plot is expected to be dependent upon both the specific sample and experimental conditions, which we consider in Appendix B by considering two other scenarios using different sets of realistic device parameters. In both cases, we find that the plots are qualitatively similar with some variation in the shape of the green region; however, the sensitivity to the bias-light conditions is very similar in each case suggesting that the criterion $V_{\mathrm{OC}} / V_{\mathrm{OC}}(1$ Sun) $>0.5$ has good generality for the validity of the small-perturbation approximation. Acceptable ranges for the excitation-pulse fluence are less consistent, but we are able to suggest a conservative value of $\Delta V_{0} / V_{\mathrm{OC}}(1$ Sun $<0.2$, which lies within the green region in each case. We note that other studies have suggested alternative criteria for determining the small-perturbation regime; for comparison, the shaded area in Fig. 6(c) satisfies the criterion that $\Delta V_{0}<\left(5 \%\right.$ of $\left.V_{0}\right)$. The criterion, $\Delta V_{0}<10 \mathrm{mV}$ covers a smaller region within this shaded area but is too small to see clearly on this scale - an indication of its limited practicability. Importantly the $\Delta V_{0}<\left(5 \%\right.$ of $\left.V_{0}\right)$ criterion encompasses a range of $\tau_{\Delta V} / \tau_{\Delta n}$ values from 0.94 to 1.94 , but covers only a small portion of the green area on the plot corresponding to this level of accuracy $\left(0.9<\tau_{\Delta V} / \tau_{\Delta n}<2.0\right)$ and so is unnecessarily restrictive. Since the shaded region is the area previously accepted as the valid small-perturbation regime [4], this means that much larger excitation fluences can be used resulting in improved experimental signal-to-noise ratio with no detriment to the accuracy of the data.

\section{Differential capacitance method (evaluating $\boldsymbol{n}$ )}

Evaluation of the charge-carrier density in the sample is achieved using the differential capacitance method, using Eq. (3) to estimate the capacitance of the device as a function of the open-circuit voltage under different bias conditions. The accuracy of this method is dependent upon the accuracy of the input parameters $\Delta Q$ and $\Delta V_{0}$ (discussed above). It also assumes the small-perturbation assumption, such that $\left(\Delta Q / \Delta V_{0}\right) \approx(\partial Q / \partial V)$.

The photovoltaic device is expected to have a chemical capacitance, with an exponential dependence on voltage, and a fixed geometric capacitance, as represented by Eq. (8). Both of these elements are included in the simulation and values obtained for the differential capacitance show a good fit with Eq. (4), as demonstrated in Fig. 4(b). However, it is important to note that the fixed capacitance value, $D$, obtained from this fitting $(64.5 \pm 1.1 \mathrm{nF})$ is almost an order of magnitude larger than the geometric capacitance in the model (parallel-plate capacitance of $10 \mathrm{nF}$ ). For this reason it is described as an "effective fixed capacitance." To understand the origin of this effect, we consider the accuracy of the differential capacitance method and its reliance on the small-perturbation approximation.

Starting from Eq. (8), we are able to compare expressions for both the derivative of stored charge $Q$ with respect to $V$ and the gradient calculated from a finite difference $\Delta V_{0}$ :

$$
\begin{gathered}
Q=A e d n_{i} \exp (\gamma V)+\frac{A \varepsilon V}{d}, \\
\frac{\partial Q}{\partial V}=\gamma A e d n_{i} \exp (\gamma V)+\frac{A \varepsilon}{d}, \\
\frac{\Delta Q}{\Delta V_{0}} \equiv \frac{Q\left(V+\Delta V_{0}\right)-Q(V)}{\Delta V_{0}} \\
=\frac{A e n_{i} d}{\Delta V_{0}} \exp (\gamma V)\left[\exp \left(\gamma \Delta V_{0}\right)-1\right]+\frac{A \varepsilon}{d},
\end{gathered}
$$

where $\varepsilon$ is the (absolute) permittivity of the active layer.

Figure 7(a) compares the differential capacitance values extracted from the simulated TPV data using the method outlined above with the expressions given in Eqs. (9) and (10). We consider Eq. (9) to represent the expected capacitance of the device and it is clear that extracted values provide a systematic overestimate, which is most obvious for low-intensity bias conditions. The main reason for this discrepancy is that $\Delta Q$ is not sufficiently small with respect to $Q$ such that the approximation $\left(\Delta Q / \Delta V_{0}\right) \approx(\partial Q / \partial V)$ fails. Eq. (10) corrects for the failure of this approximation, 
which we demonstrate by evaluating it using the expected $\Delta V_{0}$ and $V_{0}$ values taken from the simulated TPV results and plotting it in Fig. 7(a). We find that this equation reproduces the extracted values of differential capacitance much better than Eq. (9), although still not perfectly. This imperfection indicates the presence of additional, although weaker, effects that have not been addressed; we suggest that these probably arise from the evaluation of $\Delta V_{0}$ based on the pseudo-first-order assumption discussed above.

The goal of the differential charging method is to evaluate the charge-carrier density in the bulk of the device by integrating the chemical capacitance up to the appropriate open-circuit voltage. The accuracy of this method is reliant on choosing an appropriate function for fitting the differential capacitance values. Figure 7(b) compares the extracted charge-carrier densities from the simulated TPV measurement data with the expected values taken directly from the simulation. Here we consider three different options for extracting the charge-carrier density from the measured differential capacitance: (i) fitting the capacitance with an exponential chemical capacitance and a fixed geometric capacitance, as in Eq. (9); (ii) adjusting the measured differential capacitance using calculated values for the ratio $\left(\Delta Q / \Delta V_{0}\right) \approx(\partial Q / \partial V)$ and then fitting with Eq. (9) (note that, while this case provides an instructive comparator, it is not experimentally useable since it requires prior knowledge of the device parameters); and (iii) fitting the capacitance with an unconstrained effective fixed capacitance, $D$, as in Eq. (4).

Comparison of the resulting charge-carrier densities shows that (i) overestimates $n$ by up to $70 \%$ for the lowest values of $V_{\mathrm{OC}}$, whereas (ii) gives a closer underestimate, within $15 \%$ of the expected value. However, the best estimate is given using the effective-fixed-capacitance correction, $D$, which is within $10 \%$ of the expected value over the range considered here. The physical interpretation of $D$ is not obvious but the correction applied in case (ii) reveals that the failure of the small-perturbation approximation does result in a curved plot in Fig. 7(a), which is comparable to the effect of an additional fixed capacitance. We therefore conclude that the inclusion of $D$ acts as a first-order correction for divergence from the small-perturbation regime. The empirical effectiveness of introducing this correction and the simplicity of its implementation compared with the other corrections considered here suggests that this is an appropriate and suitable approximation for improving the accuracy of experimental TPV measurements.

The goal of a typical TPV measurement is to elucidate the charge recombination order in a photovoltaic device by considering the relationship between the charge-carrier lifetime $\tau_{\Delta n}$ and bulk charge-carrier density $n$, expecting to discover a power-law relationship that can be fitted with Eq. (6). The discussion above has considered a number of factors relating to the analysis of TPV data that
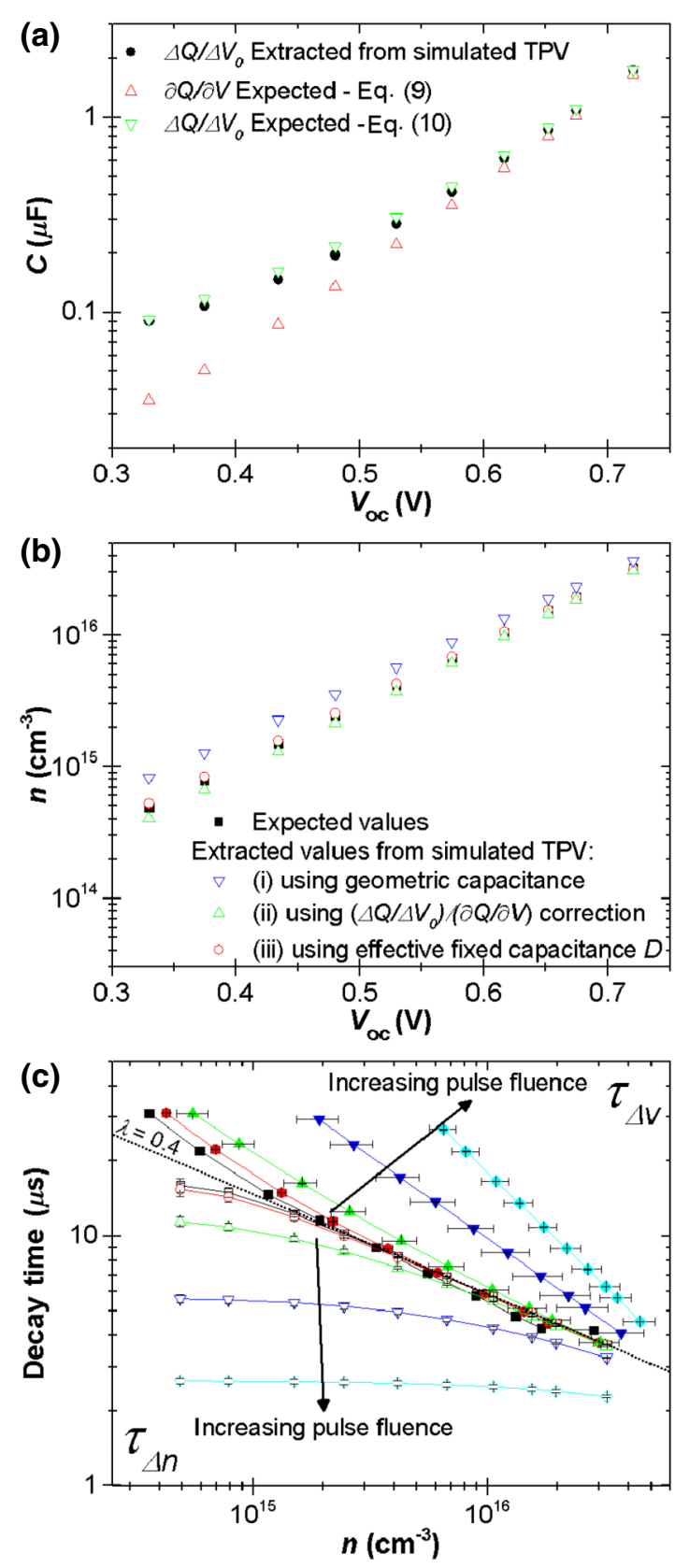

FIG. 7. (a) Differential capacitance values for the simulation shown in Fig. 4 comparing the extracted $\Delta Q / \Delta V_{0}$ (filled circles) values from analysis of the simulated TPV and TPC results with expected values for $\partial Q / \partial V$ (filled triangles) and $\Delta Q / \Delta V_{0}$ (open triangles) based on Eqs. (9) and (10). (b) Bulk chargecarrier density plotted against $V_{\mathrm{OC}}$ comparing expected values taken directly from the simulation with extracted values derived from the simulated data applying corrections (i), (ii), and (iii). (c) Comparison of TPV decay time $\tau_{\Delta V}$ plotted against chargecarrier density values derived from analysis of the TPV simulation with charge-carrier-density decay time $\tau_{\Delta n}$ plotted against expected charge-carrier-density values taken directly from the simulation, for excitation laser fluences with each order of magnitude over the approximate range $1 \mu \mathrm{J} \mathrm{m}^{-2}$ to $10 \mathrm{~mJ} \mathrm{~m}^{-2}$. The dotted line indicates the power-law relationship expected from the simulation parameters. 


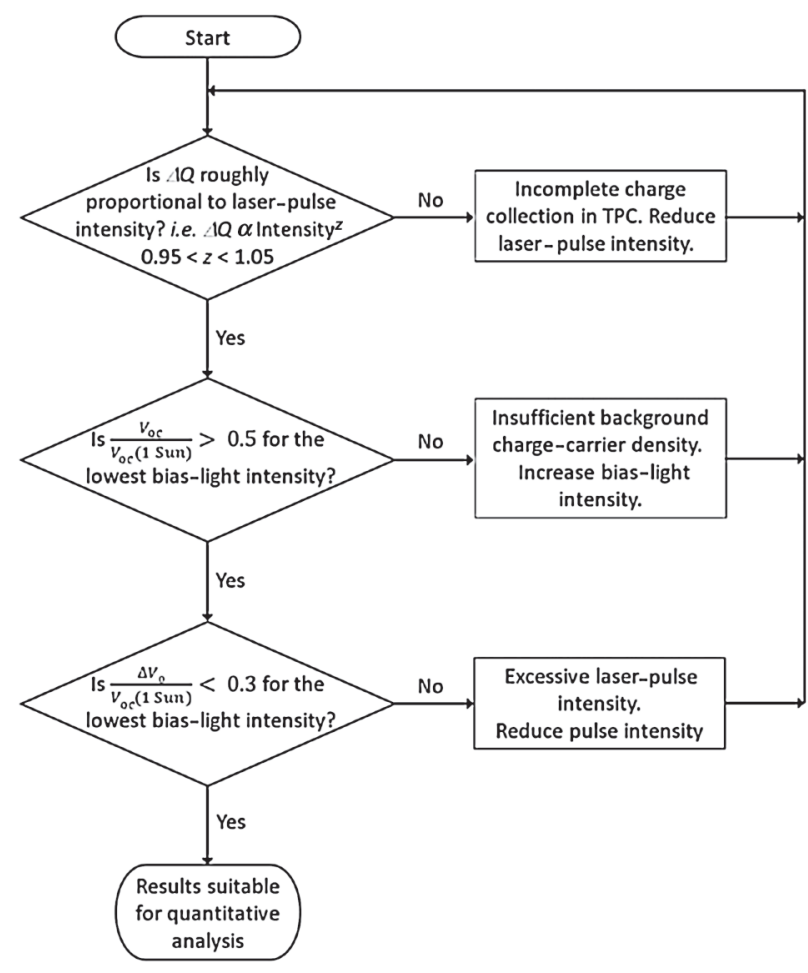

FIG. 8. Proposed flowchart for assessing whether experimental TPV and TPC measurements are suitable for quantitative analysis using the techniques described in this article.

affect the accuracy with which the results can be interpreted. In general, the method is heavily reliant on the small-perturbation approximation, and so significant deviations from this regime are problematic and arise when the intensity of the pulsed excitation is too high and/or the bias-light intensity too low. Figure 7(c) summarizes these effects by comparing $\tau_{\Delta V}(n)$ (where both $\tau_{\Delta V}$ and $n$ are extracted from the simulated TPV data according to the method discussed) with $\tau_{\Delta n}(n)$ (where both $\tau_{\Delta n}$ and $n$ are expected values taken directly from the simulation) for a range of excitation-pulse fluences from $1 \mu \mathrm{J} \mathrm{m}^{-2}$ to $10 \mathrm{~mJ} \mathrm{~m}^{-2}$. For low excitation fluences, both $\tau_{\Delta n}(n)$ and $\tau_{\Delta V}(n)$ converge on the expected power law where $\lambda=0.4$; however, even in this case, the plots diverge at low charge-carrier densities (corresponding to low biaslight intensities). As the excitation fluence increases, the divergence becomes more pronounced with $\tau_{\Delta n}(n)$ giving progressively shorter decay times and $\tau_{\Delta V}(n)$ giving longer decay times. In both cases, this divergence arises from the failure of the small-perturbation approximation as the transients cease to follow a monoexponential decay. For $\tau_{\Delta n}(n)$ a large perturbation $(\Delta Q)$ results in a significant increase in charge density from the equilibrium value and so charge recombination is correspondingly faster, whereas for $\tau_{\Delta V}(n), V_{\mathrm{OC}}$ follows a logarithmic relationship with respect to the charge-carrier density such that the decay in charge density resulting from a large perturbation exhibits a slower decay in the photovoltage.

\section{CONCLUSION}

This exploration into the validity of the conventional method of analyzing TPV data to extract parameters relating to the charge recombination dynamics has given rise to a number of observations, corrections, and qualifications, which are expected to have broad applicability to such measurements. In particular, we have introduced the "effective fixed capacitance" $D$ into Eq. (4) and shown that this modification significantly improves the accuracy of results extracted from TPV experiments on organic solar cells. Figure 8 attempts to summarize the findings of this work with a view to providing a flowchart for ensuring the validity of experimental results. We note that the criteria derived from this work are derived from a limited number of simulated devices, which it is reasonable to consider as representative, although it is not possible to claim that these results are fully general. Applying this process to the simulated data considered in this manuscript leads to a value for the charge-carrier recombination order of 1.48 , which is within $10 \%$ of the simulation input value of 1.40. Efforts have been undertaken to ensure that the suggested criteria can be established by an experimentalist with no a priori knowledge of the sample parameter values. Our intention is that measurements lying within these guidelines can be considered reasonable, whereas measurements outside them should be treated with skepticism. We have also shown that the proposed criteria expand the valid range of experimental parameters towards "large perturbations" resulting in improved signal-to-noise ratio and faster measurements.

\section{ACKNOWLEDGMENTS}

This work was funded by the Department for Business, Energy and Industrial Strategy (BEIS) through the National Measurement System as part of the Innovation, Research and Development program, and through the European Metrology Programme for Innovation and Research (EMPIR) Project 16ENG03-HyMet. The EMPIR initiative is co-funded by the European Union's Horizon 2020 research and innovation programme and the EMPIR participating States. Materials were supplied by Merck Chemicals Ltd. Thanks are also due to Stephen Giblin for providing a constructive review of this manuscript.

\section{APPENDIX A: DETAILED DESCRIPTION OF MODEL}

In thin-film devices, the ratio of the lateral to vertical dimensions of the active layer can be as high as $10^{6}$. Under such conditions, a full three-dimensional device simulation is so inefficient that it becomes computationally 


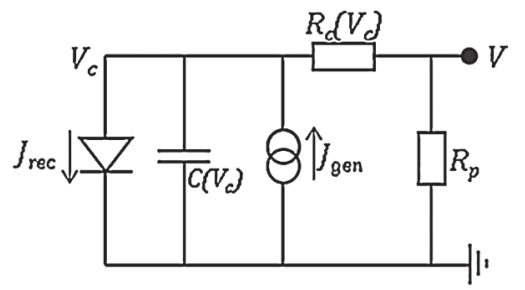

FIG. 9. Equivalent circuit diagram representing the onedimensional model used for calculating local current density through the active layer.

unworkable. Instead, it has been demonstrated that reliable results can be achieved by simulating devices with a two-dimensional simulation of transport within electrodes coupled to multiple one-dimensional models of vertical transport through the thin film [22-24]. The same principle is used here, with the exception that here we implement a transient simulation instead of the steady-state implementation used in the above references.

\section{Lateral conduction within electrodes}

Here, the term "electrode" is used to refer to the conductive layers at the top and bottom of a device stack. In the example used in this article, the bottom electrode is ITO, while the top electrode is an Ag film. However, in general the electrodes can include hole/electron transport layers, transparent conductive oxides, printed metal electrodes, or a combination of the above. Assuming that the device electrodes are planar ohmic conductors, the vector of lateral current density flowing in the bottom electrode is given by $J_{0}=-\nabla V_{0}(x, y) / R_{0}(x, y)$, where $V_{0}(x, y)$ is the local voltage on the bottom electrode at the position $(x, y)$, and $R_{0}$ $(x, y)$ is the local sheet resistance of the electrode. Where two parallel electrodes are separated by an insulator, capacitive charge is stored at the surface of the electrodes at a rate given by $(\partial \rho / \partial t)=-\nabla \cdot J_{0}=\nabla \cdot J_{1}$, where $\rho(x, y)$ is the local charge density and $J_{1}$ is the lateral current density on the top electrode. A change in the stored charge leads to a change in the voltage drop across the insulator giving $(\partial / \partial t)\left(V_{0}-V_{1}\right)=(d / \varepsilon)(\partial \rho / \partial t)$, where $V_{1}(x, y)$ is the local voltage on the top electrode, $d$ is the insulator thickness, and $\varepsilon$ is the permittivity. In the case of a thin-film device, there is an additional vertical displacement current caused by charge flowing within the active layer, whose density is represented by $J_{\text {disp }}(x, y)$. By conservation of current, these combine to give

$$
\begin{aligned}
\frac{\partial}{\partial t}\left(V_{0}-V_{1}\right) & =\frac{d}{\varepsilon}\left(\nabla \cdot \frac{\nabla V_{0}}{R_{0}}-J_{\text {disp }}\right) \\
& =\frac{d}{\varepsilon}\left(-\nabla \cdot \frac{\nabla V_{1}}{R_{1}}-J_{\text {disp }}\right) .
\end{aligned}
$$

This is solved using an FEM approach, with the additional boundary condition of fixed voltage at contact points on the top and bottom electrodes in the case of TPC, with an implicit Euler iteration scheme to simulate the device and current voltage as a function of time. The input to the FEM solution, $J_{\text {disp }}(x, y)$, is calculated at each node in the FEM grid using a time-dependent one-dimensional device model described below.

\section{Vertical transport through film}

The experimental observations represented by Eqs. (1) and (4) (that the transient photovoltage decays exponentially with time and that the capacitance grows exponentially with open-circuit voltage) have been demonstrated to hold true (for a range of voltages) in many different photovoltaic materials. Furthermore, they originate from a simple and reasonable physical model. Our aim is to derive

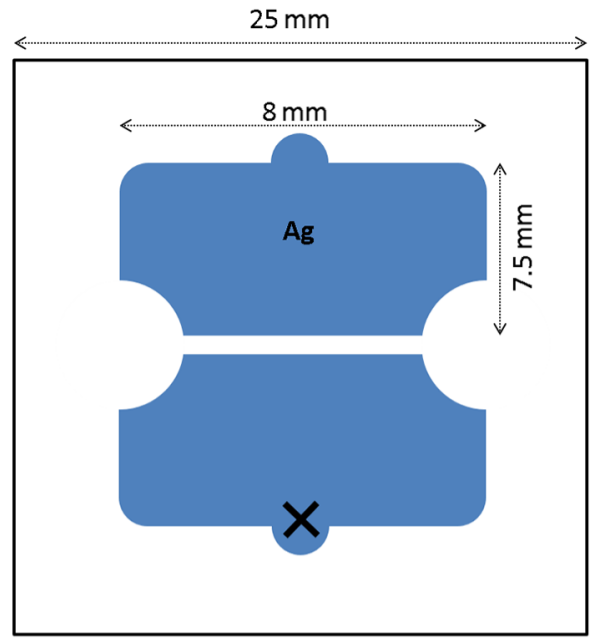

FIG. 10. Diagram showing the geometries of the ITO and Ag electrodes considered in this study. The Ag electrodes define two cells with an area of $1 \mathrm{~cm}^{2}$ each, but only the lower cell is connected. Crosses mark the positions of electrical connections. 
TABLE III. Simulation parameters for transient responses used in this study.

\begin{tabular}{|c|c|c|c|c|c|c|c|c|}
\hline Step & Duration (s) & $\begin{array}{l}\text { Steady } \\
\text { state? }\end{array}$ & Voltage (V) & $\begin{array}{l}\text { Background charge } \\
\text { generation }\left(\mathrm{m}^{-3} \mathrm{~s}^{-1}\right)\end{array}$ & $\begin{array}{l}\text { Laser charge } \\
\text { generation }\left(\mathrm{m}^{-3} \mathrm{~s}^{-1}\right)\end{array}$ & $\begin{array}{l}\text { Laser } x \\
\text { position (m) }\end{array}$ & $\begin{array}{l}\text { Laser } y \\
\text { position (m) }\end{array}$ & $\begin{array}{l}\text { Laser } \\
\text { radius }(\mathrm{m})\end{array}$ \\
\hline 1 & $1 \times 10^{-4}$ & Yes & 0 & $2.25 \times 10^{27}$ & 0 & 0 & 0 & 0 \\
\hline 2 & $5 \times 10^{-9}$ & No & 0 & $2.25 \times 10^{27}$ & $1.00 \times 10^{30}$ & 0.0125 & 0.0125 & 0.0125 \\
\hline 3 & $1 \times 10^{-8}$ & No & 0 & $2.25 \times 10^{27}$ & 0 & 0 & 0 & 0 \\
\hline 4 & $1 \times 10^{-4}$ & No & 0 & $2.25 \times 10^{27}$ & 0 & 0 & 0 & 0 \\
\hline
\end{tabular}

a simple one-dimensional device model that is faithful to this behavior.

The simplest and most common representation for a photovoltaic device is the one-diode model. It can be adjusted to exhibit the observed transient behavior with the simple addition of a voltage-dependent capacitance, as shown in Fig. 9.

The constant-current element represents photogenerated current, which has a current density of $J_{\text {gen }}=G e d$, where $G$ is the charge-carrier generation rate per unit volume in the active layer, $e$ is the elementary charge, and $d$ is the active layer thickness. The voltage-dependent capacitor $C\left(V_{c}\right)$ represents the chemical capacitance of the active layer. It can be expressed in terms of the carrier density $C\left(V_{c}\right)=e n_{i} \gamma d \exp \left(\gamma V_{c}\right)$ (per unit area), where $n_{i}$ is the intrinsic carrier density, $V_{c}$ represents the chemical potential (Fermi-level splitting) of the active layer, and $\gamma$ is an empirically determined exponential constant related to the carrier-density ideality factor: $\alpha=e /\left(2 \gamma k_{B} T\right)$, where $k_{B}$ is the Boltzmann constant and $T$ is temperature. This model relies on the assumption that positive and negative charge-carrier densities are balanced
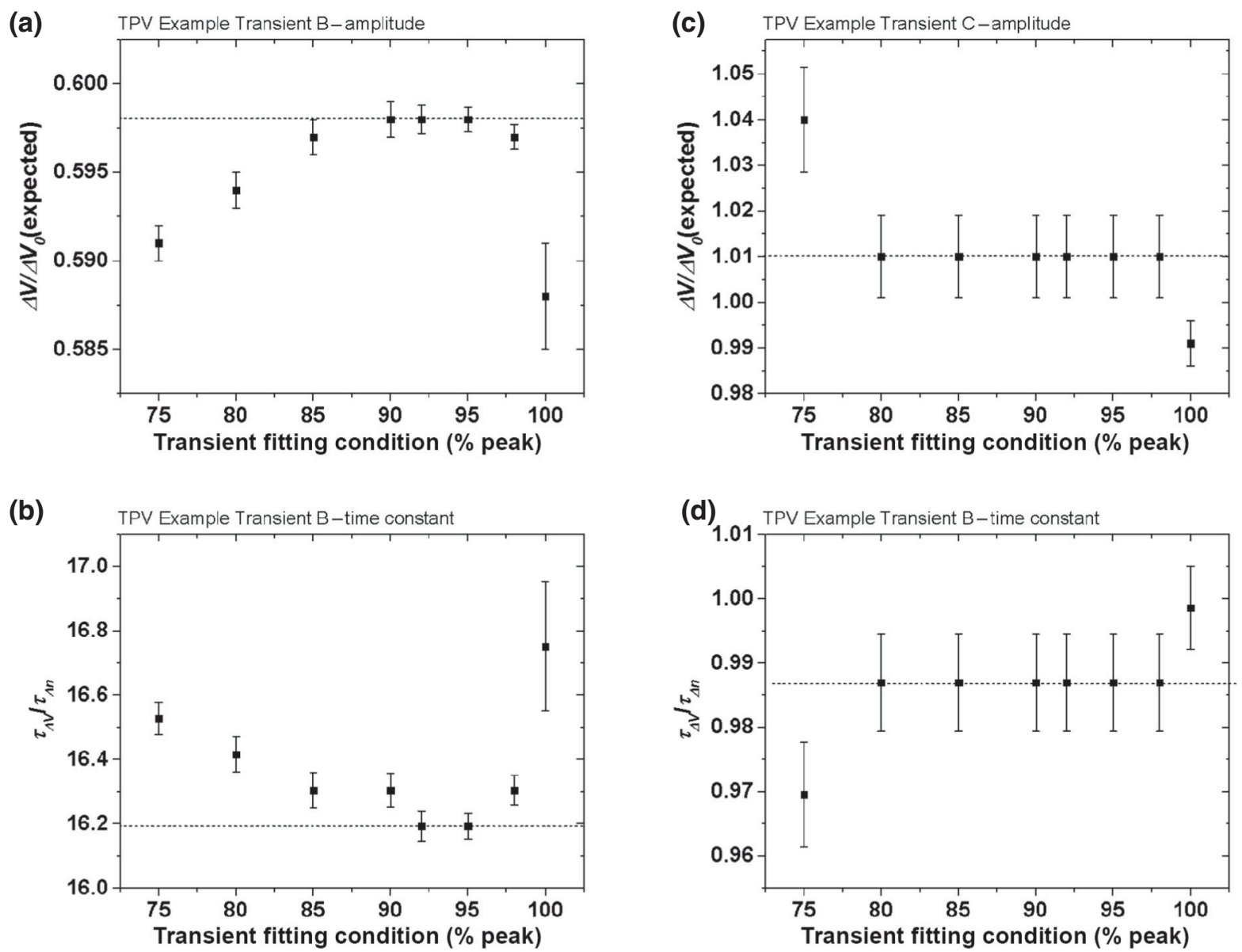

FIG. 11. Comparison of fitting parameters extracted from simulated TPV results using transients $B$ and $C$ from Fig. 6(b) as examples, where exponential fitting is applied to the decay tail staring from $75 \%$ up to $100 \%$ of the peak value. (a)-(c) show the fitted TPV amplitude $\Delta V$, normalized to the expected value for $B$ and $C$, respectively. (b)-(d) show the fitted TPV time constant $\tau_{\Delta V}$, normalized to the time constant of the charge-carrier density for $B$ and $C$, respectively. 
(a)
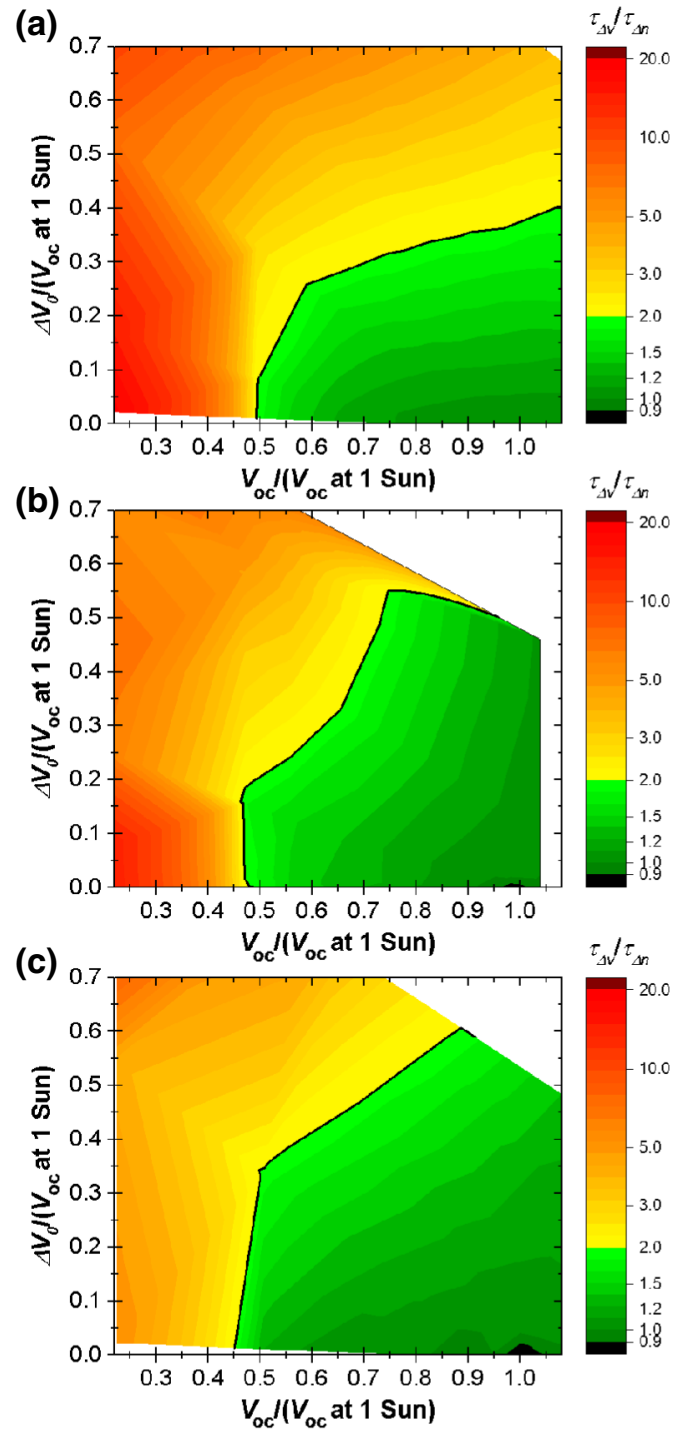

FIG. 12. Color plots showing the ratio $\tau_{\Delta V} / \tau_{\Delta n}$ plotted against $\Delta V_{0} /\left(V_{\mathrm{OC}}\right.$ at $\left.1 \mathrm{Sun}\right)$ and $V_{\mathrm{OC}} /\left(V_{\mathrm{OC}}\right.$ at $\left.1 \mathrm{Sun}\right)$ for three different sets of device parameters. The green region shows the measurement range where the ratio lies between 0.9 and 2.0. White areas indicate an absence of data points. (a) reproduces the case considered in the main manuscript and parameters for (a)-(c) are compared in Table IV.

within the bulk of the active layer deriving from a charge-carrier density of $n=n_{i} \exp \left(\gamma V_{c}\right)$. Charge-carrier recombination occurs according to the power-law behavior $(\partial n / \partial t)=k_{\mathrm{br}} n_{i}^{2}\left[1-\left(n / n_{i}\right)^{\lambda+1}\right]$, where we refer to the rate $k_{\mathrm{br}}$ as the effective bimolecular-recombination rate coefficient. Substituting in the dependence of carrier density on voltage gives a recombination current density $J_{\text {rec }}=e L_{z} k_{\mathrm{br}} n_{i}^{2}\left\{\exp \left[(\lambda+1) \gamma V_{c}\right]-1\right\}$, which is represented by the diode in Fig. 9. Finally, the resistor $R_{c}$ represents the loss of potential required to transport charge carriers through the active layer. Two sources of current are considered: firstly, the motion of bulk charge carriers under the external potential; and secondly, the injection of charge carriers into the bulk. Charge is driven by the electrochemical potential difference $V-V_{c}$ between the externally applied voltage and the build-up of charge carriers within the device. Assuming a constant effective mobility $\mu$, the current density is given by $J_{c}=(e \mu / d)\left(V-V_{c}\right)\left(n+n_{\text {inj }}\right)$ and we assume the injected charge density follows $n_{\text {inj }}=n_{i}$ $\exp \left[\gamma\left(V-V_{c}\right)\right]$. Note that under the conditions of perfect bimolecular recombination $(\lambda=1)$, an ideal density of states $(\alpha=1)$, and a high mobility $(\mu \rightarrow \infty)$, the equivalent circuit tends to a perfect single-diode model.

Using the above model, the FEM simulation is completed by evaluating the following equations for each node in the FEM model at each time step:

$$
\begin{aligned}
J_{\text {disp }}= & \frac{e \mu}{d}\left(V-V_{c}\right)\left\{n_{i} \exp \left(\gamma V_{c}\right)+n_{i} \exp \left[\gamma\left(V-V_{c}\right)\right]\right\} \\
& +V / R_{p}, \\
\frac{\partial V_{c}}{\partial t} & =\gamma^{-1} \exp \left(\gamma V_{c}\right)\left(J_{c}+J_{\text {gen }}-J_{\text {rec }}\right) / n_{i} e d .
\end{aligned}
$$

\section{Simulation conditions}

The architecture of the device is defined by the electrodes whose geometries are illustrated in Fig. 10.

The FEM simulation first converts the cell geometry into a mesh. We find that approximately 2000 mesh points is sufficient for the solution to converge. Pulsed optical excitation is simulated using the sequence of steps listed in Table III. The background and laser charge-generation rates vary corresponding to different bias-light intensities and excitation laser fluences, respectively. The values shown here approximate to a bias-light intensity of 0.75 Sun and a laser-pulse fluence of $100 \mu \mathrm{J} \mathrm{m}^{-2}$. Positions in the simulation are measured relative to the bottom left-hand corner of the device.

The initial steady-state solution is found with a tolerance of $1 \mu \mathrm{V}$. During the simulation, the maximum time step is $1 / 1000$ th of the step duration and the maximum transient voltage step is $1 \mathrm{mV}$. The temperature is considered to remain at $300 \mathrm{~K}$ throughout.

\section{APPENDIX B: SUPPLEMENTARY DISCUSSION OF RESULTS}

Figure 11 considers the fitting of the TPV signal with a single exponential decay. In the manuscript we state that satisfactory fitting requires that the initial rise and early development of the transient is neglected, and fitting from $95 \%$ of the peak value is found to be a reliable condition. Here we consider how the extracted fitting parameters are affected by varying this condition from $75 \%$ up to $100 \%$ of the peak TPV signal. Using the simulated transients $B$ and $C$ from Fig. 6(b) as examples, Fig. 11 shows the fitted amplitudes and transient time constants of the TPV signals. 
TABLE IV. Simulated device parameters for cases (b) and (c) corresponding to plots in Fig. 12.

\begin{tabular}{llll}
\hline \hline & Case (a) & Case (b) & Case (c) \\
\hline Active layer thickness $(\mathrm{nm})$ & 300 & 100 & 300 \\
Active layer area $\left(\mathrm{cm}^{2}\right)$ & 1 & 1 & 1 \\
Relative permittivity & 3.5 & 3.5 & 3.5 \\
ITO electrode sheet resistance $\left(\Omega \square^{-1}\right)$ & 15 & 15 & 15 \\
Ag electrode sheet resistance $\left(\Omega \square^{-1}\right)$ & 0.1 & 0.1 & 0.1 \\
Shunt resistivity $(\Omega \mathrm{m})$ & $1 \times 10^{8}$ & $1 \times 10^{7}$ & $1 \times 10^{8}$ \\
Intrinsic charge-carrier density $\left(\mathrm{m}^{-3}\right)$ & $1.4 \times 10^{19}$ & $7 \times 10^{19}$ & $1 \times 10^{19}$ \\
Carrier-density ideality factor & 1.8 & 2 & 1.8 \\
Effective charge-carrier mobility $\left(\mathrm{m}^{2} \mathrm{~V}^{-1} \mathrm{~s}^{-1}\right)$ & $5 \times 10^{-8}$ & $5 \times 10^{-9}$ & $5 \times 10^{-7}$ \\
Charge-carrier recombination order & 1.4 & 1.9 & 1.8 \\
Effective bimolecular recombination rate $\left(\mathrm{m}^{3} \mathrm{~s}^{-1}\right)$ & $6.3 \times 10^{-16}$ & $1 \times 10^{-17}$ & $1 \times 10^{-16}$ \\
\hline \hline
\end{tabular}

In each case the values are normalized to the expected values resulting in trends with optimum values where the fitting is nearest to the expected value. Across all of these cases, accurate values are obtained from the fitting with a criterion of up to $95 \%$, which we therefore adopt as a recommended value. Note that point $B$ corresponds to a region in Fig. 6(a) for which the small-perturbation approximation is not considered valid, whereas point $C$ is within the "acceptable" region.

Figure 12 considers the relationship between the ratio $\tau_{\Delta V} / \tau_{\Delta n}$ and the experimental conditions represented by $\Delta V_{0} /\left(V_{\mathrm{OC}}\right.$ at $\left.1 \mathrm{Sun}\right)$ and $V_{\mathrm{OC}} /\left(V_{\mathrm{OC}}\right.$ at 1 Sun $)$ for different sets of device parameters: cases (a), (b), and (c) are as presented in Table IV. Only case (a) is considered in the main manuscript [Fig. 6(c)] but it is not possible to exhaustively demonstrate the generality of the validity criteria derived from those results. Here we explore the alternative cases (b) and (c), using different parameters in the simulation. There are significant differences in the results plotted for each case; however, we note that the general form of these plots is the same and that the approximate "size" of the accepted parameter space (plotted in green) is comparable in each case. This result indicates that the discussion in this manuscript and the suggested validity criteria are not unique to a single set of device parameters and can be expected to apply with a reasonable level of generality.

[1] A. Cuevas and R. A. Sinton, in Practical Handbook of Photovoltaics: Fundamentals and Applications, edited by T. Markvart and L. Castañer (Elsevier, Oxford, UK, 2003), p. 227-252.

[2] P. R. F. Barnes, K. Miettunen, X. Li, A. Y. Anderson, T. Bessho, M. Gratzel, and B. C. O'Regan, Interpretation of optoelectronic transient and charge extraction measurements in dye-sensitized solar cells, Adv. Mater. 25, 1881 (2013).

[3] A. B. Walker, L. M. Peter, K. Lobato, and P. J. Cameron, Analysis of photovoltage decay transients in dye-sensitized solar cells, J. Phys. Chem. B 110, 25504 (2006).
[4] Z. Li, F. Gao, N. C. Greenham, and C. R. McNeill, Comparison of the operation of polymer/fullerene, polymer/polymer, and polymer/nanocrystal solar cells: A transient photocurrent and photovoltage study, Adv. Funct. Mater. 21, 1419 (2011).

[5] D. Credgington and J. R. Durrant, Insights from transient optoelectronic analyses on the open-circuit voltage of organic solar cells, J. Phys. Chem. Lett. 3, 1465 (2012).

[6] R. C. I. Mackenzie, C. G. Shuttle, G. F. Dibb, N. Treat, E. Von Hauff, M. J. Robb, C. J. Hawker, M. L. Chabinyc, and J. Nelson, Interpreting the density of states extracted from organic solar cells using transient photocurrent measurements, J. Phys. Chem. C 117, 12407 (2013).

[7] C. G. Shuttle, R. Hamilton, B. C. O'Regan, J. Nelson, and J. R. Durrant, Charge-density-based analysis of the current-voltage response of polythiophene/fullerene photovoltaic devices, Proc. Natl. Acad. Sci. U.S.A. 107, 16448 (2010).

[8] C. G. Shuttle, B. O’Regan, A. M. Ballantyne, J. Nelson, D. D. C. Bradley, J. De Mello, and J. R. Durrant, Charge extraction analysis of charge carrier densities in a polythiophene/fullerene solar cell: Analysis of the origin of the device dark current, Appl. Phys. Lett. 92, 3 (2008).

[9] B. C. O’Regan, P. R. F. Barnes, X. Li, C. Law, E. Palomares, and J. M. Marin-Beloqui, Optoelectronic studies of methylammonium lead iodide perovskite solar cells with mesoporous $\mathrm{TiO}_{2}$ : Separation of electronic and chemical charge storage, understanding two recombination lifetimes, and the evolution of band offsets during $\mathrm{J}-\mathrm{V}$ hysteresis, J. Am. Chem. Soc. 137, 5087 (2015).

[10] C. G. Shuttle, A. Maurano, R. Hamilton, B. O’Regan, J. C. De Mello, J. R. Durrant, B. O'Regan, J. C. De Mello, and J. R. Durrant, Charge extraction analysis of charge carrier densities in a polythiophene/fullerene solar cell: Analysis of the origin of the device dark current, Appl. Phys. Lett. 93, 183501 (2008).

[11] W. Zhao, D. Qian, S. Zhang, S. Li, O. Inganäs, F. Gao, and J. Hou, Fullerene-free polymer solar cells with over $11 \%$ efficiency and excellent thermal stability, Adv. Mater. 28, 4734 (2016).

[12] Z. He, B. Xiao, F. Liu, H. Wu, Y. Yang, S. Xiao, C. Wang, T. P. Russell, and Y. Cao, Single-junction polymer solar cells with high efficiency and photovoltage, Nat. Photonics 9, 174 (2015). 
[13] H. Zhang, H. Yao, W. Zhao, L. Ye, and J. Hou, Highefficiency polymer solar cells enabled by environmentfriendly single-solvent processing, Adv. Energy Mater. 6, 1502177 (2016).

[14] S. Hong, H. Kang, G. Kim, S. Lee, S. Kim, J.-H. Lee, J. Lee, M. Yi, J. Kim, H. Back, J.-R. Kim, and K. Lee, A series connection architecture for large-area organic photovoltaic modules with a $7.5 \%$ module efficiency, Nat. Commun. 7 , 1 (2016).

[15] B. C. O’Regan, K. Bakker, J. Kroeze, H. Smit, P. Sommeling, and J. R. Durrant, Measuring charge transport from transient PHotovoltage rise times. A new tool to investigate electron transport in nanoparticle films, J. Phys. Chem. B 110, 17155 (2006).

[16] L. C. C. Elliott, J. I. Basham, K. P. Pernstich, P. R. Shrestha, L. J. Richter, D. M. Delongchamp, and D. J. Gundlach, Probing charge recombination dynamics in organic photovoltaic devices under open-circuit conditions, Adv. Energy Mater. 4, 1 (2014).

[17] S. Wood, D. O'Connor, C. W. Jones, J. D. Claverley, J. C. Blakesley, C. Giusca, and F. A. Castro, Transient photocurrent and photovoltage mapping for characterisation of defects in organic photovoltaics, Sol. Energy Mater. Sol. Cells 161, 89 (2017).

[18] S. Berny, N. Blouin, A. Distler, H. J. Egelhaaf, M. Krompiec, A. Lohr, O. R. Lozman, G. E. Morse, L. Nanson, A. Pron, T. Sauermann, N. Seidler, S. Tierney,
P. Tiwana, M. Wagner, and H. Wilson, Solar trees: First large-scale demonstration of fully solution coated, semitransparent, flexible organic photovoltaic modules, Adv. Sci. 3, 1 (2015).

[19] MATLAB R2015b (The MathWorks Inc., Natick, MA, 2015).

[20] A. Köhler and H. Bässler, Electronic Processes in Organic Semiconductors: An Introduction (WILEY-VCH, Weinheim, Germany, 2015).

[21] D. Baran, T. Kirchartz, S. Wheeler, S. Dimitrov, M. Abdelsamie, J. Gorman, R. Ashraf, S. Holliday, A. Wadsworth, N. Gasparini, P. Kaienburg, H. Yan, A. Amassian, C. J. Brabec, J. Durrant, and I. McCulloch, Reduced voltage losses yield 10\% efficient fullerene free organic solar cells with $>1 \mathrm{~V}$ open circuit voltages, Energy Environ. Sci. 9, 3783 (2016).

[22] U. Malm and M. Edoff, Simulating material inhomogeneities and defects in CIGS thin-film solar cells, Prog. Photovolt. Res. Appl. 17, 306 (2009).

[23] T. Lanz, M. Bonmarin, M. Stuckelberger, C. Schlumpf, C. Ballif, and B. Ruhstaller, Electrothermal finite-element modeling for defect characterization in thin-film silicon solar modules, IEEE J. Sel. Top. Quantum Electron. 19, 1 (2013).

[24] X. Sun, J. Raguse, R. Garris, C. Deline, T. Silverman, and M. A. Alam, in 2015 IEEE 42nd Photovolt. Spec. Conf. (2015). 